\title{
Asymmetrically Wettability Janus Cotton Fabrics Fabricated via Interfacial Ion Migration for Surfactant-stabilized Oil-in-water Emulsions
}

\section{Pu Yang}

Southwest University

Ruimin Hu

Southwest University

Bin Yu

Southwest University

Yiwei Sun

Southwest University

Yiping Liu

Southwest University

Ming Lu ( $\square$ lumingswu@126.com)

Southwest University https://orcid.org/0000-0002-9360-473X

\section{Research Article}

Keywords: Cotton fabric, Janus membrane, asymmetrical wettability, emulsion separation

Posted Date: March 1st, 2021

DOI: https://doi.org/10.21203/rs.3.rs-232628/v1

License: (1) This work is licensed under a Creative Commons Attribution 4.0 International License. Read Full License 


\section{Abstract}

Separation of surfactant-stabilized oil-water emulsions seems to be challenging owing to its diverse repercussions on environment and human life. The asymmetrical wettability Janus cotton fabric (JMH@CF) with high separation performance was prepared by two-step method, which related to interfacial ion migration technology and unilateral spraying treatment. In detail, the immobilization of magnesium hydroxide $\left(\mathrm{Mg}(\mathrm{OH})_{2}\right)$ caused the formation of the rough micro/nanostructure of cotton fabric surface, which was helpful to superhydrophilic property. Stearic acid as a coating created the unilateral superhydrophobic surface with low surface energy. J-MH@CF showed asymmetric wettability, featuring diode-like directional water transmission. Wettability, directional transmission and separation performance of J-MH@CF membrane were investigated systematically. The asymmetric wettability architecture was demonstrated to play a key role in separating surfactant-stabilized oil-in-water emulsions. Impressingly, the separation performance was not affected by the type of surfactants. For emulsion stabilized by sodium dodecyl sulfate (SDS), the separation flux driven by gravity was approximately $500 \mathrm{~L} \mathrm{~m}^{-2} \mathrm{~h}^{-1}$, and all separation efficiencies were over $99.3 \%$. CTAB/Oil/ $\mathrm{H}_{2} \mathrm{O}$ emulsion and the Tween-60/Oil/ $\mathrm{H}_{2} \mathrm{O}$ emulsion also could be successfully separated with high separation efficiency and separation flux. During the whole separation process, the oil droplets surrounded by surfactants (Oil-Ss) were difficult to demulsify and gathered on the surface of the fabric to form a "creamy layer", which was beneficial to improve separation efficiency and could be cleaned off so that J-MH@CF membrane was not contaminated. In addition, the J-MH@CF membrane exhibited robust reusability for separation, which was promising for remediation of oily wastewater containing surfactants.

\section{Introduction}

In recent years, a large volume of industrial oily wastewater containing surfactants have been produced in many fields, such as detergent, medicine, food, cosmetic, textile industries and etc., while the reactors, storage tanks and pipes have to be cleaned repeatedly (Ostertag et al. 2012; McClements and Jafari 2018). Oily wastewater has been a serious threat to the survival of aquatic animals and plants and human health. Solution of the wastewater pollution has attracted the attention of researchers.

Depending on the forming conditions, oil/water mixtures are categorized into immiscible oil/water mixtures and emulsified oil/water mixtures. Because of the thermodynamic instability, the insoluble oil/water mixtures will eventually separate, with the heavier phase sedimentating on bottom and the lighter one floating on top. On the contrary, the spontaneous phase separation is difficult to occur in oil/water emulsions containing surfactants. As conventional oil/water separation technologies, skimming, centrifugation and adsorption suffer from the limitations owing to their poor selectivity for water phase and oil phase, low separation efficiency and secondary pollution, respectively (Liu et al. 2019). And the above technologies are only suitable for the separation of immiscible oil/water mixtures, but not for the separation of oil/water emulsions. 
Janus membranes with asymmetric wettability which are considered to be a highly selective, low-cost, efficient and environment-friendly materials have exhibited an excellent superiority in oil/water emulsions separation (Panwar et al. 2018). Janus membrane with asymmetric properties on the two sides is a kind of new membrane materials, which is fabricated and designed by adjusting material's surface free energy and regulating a micro/nanostructured rough surface (Manna and Lynn 2015; Gupta et al. 2017). Resulting in special infiltration, it has been found in the study that the surface with lower surface free energy is more hydrophobic while the surface with higher surface free energy is more hydrophilic. Besides, the hydrophobicity or hydrophilicity of the membrane surface enhanced by micro-nanoscale rough structure (Ge et al. 2018).

As a result of the wettability gradient or structure gradient, the opposite properties on two sides of a Janus membrane generate the pressure gradients so that the transport of liquid in the membrane is anisotropic (Woo et al. 2020). Herein, the liquid moves across the membrane in an irreversible direction like a diode, that is, the liquid "diode" phenomenon. Compared with conventional membrane materials, the existence of "diode" phenomenon makes Janus membrane achieve higher separation efficiency and selectivity in oil/water separation (Li et al. 2016; Wang et al. 2017b). Such a "smart" property of Janus membrane with double-sided asymmetric property indicates great promise not only for oil/water separation (Liu et al. 2017b, 2020; Wang et al. 2017a; An et al. 2018; Lin et al. 2019; Yao et al. 2019; Zhang et al. 2019; Zuo et al. 2020)but also for water collection (Gao et al. 2018), functional textiles (Dai et al. 2019; Wang et al. 2020), and other field (Li et al. 2020).

Based on previous reported literatures, a feasible technology based on demulsification before the separation process has emerged. Driven by electric, hydrodynamic or other external forces, the emulsified droplets approach the surface of the membrane. After captured by the hydrophilic or hydrophobic surface, the droplets combine with each other to form larger droplets induced by wetting and shearing collisions. Then the droplets are automatically delaminated with the continuous phase under the influence of gravity and buoyancy. Wang et al. prepared a Janus fabric with a hydrophilic polymer, poly (N, N-dimethylaminoethyl methacrylate) (PDMAEMA), on one side and a hydrophobic polymer, poly (dimethyl siloxane), on the other side (Wang et al. 2016). PDMAEMA was chosen owing to it could ionize in water and aggregate emulsion droplets by screening the electrostatic repulsion. PDMS was selected due to its low surface tension, which made cotton fabric super hydrophobic. Interestingly, the Janus fabric separated oil from oil-in-water emulsions when the PDMAEMA-coated side faced the feed emulsions. The emulsified droplets strike the membrane and the coatings of membrane trigger the deemulsification of emulsions via the charge-screening effect.

By vacuum filtration, Wang et al. prepared Janus membrane using positively and negatively charged CNT coating and hydrophobic polypropylene microfiltration membrane as raw materials. De-emulsification is greatly facilitated by the fact that the charged CNT coating attracts oil droplets with opposite charges (An et al. 2018). In general, surfactants tend to adsorb on the membrane surface with opposite charges, so the membrane shows selectivity for a single ionic type of surfactant. This selective membrane is not suitable for the separation of emulsion stabilized by unspecified surfactant. In addition, the opposite 
charges contribute to de-emulsification, but aggravate the membrane fouling. As is known to all, once the oils contact the membrane, pollution cannot be avoided, resulting in blockage of surface pores and a serious decrease in flux and rejection rate. Liu et al. have proved that the charge and surface hydrophilicity of surface caused irreversible membrane fouling (Liu et al. 2017a).

Therefore, it is promising and significant to find membrane materials that are more resistant to pollution and suitable for separation of oily wastewater containing unspecified surfactants. As a biodegradable material, cotton fabric has been widely modified into a substrate for the preparation of oil-water separation membrane because of its excellent properties, including flexibility, porosity and low cost (Xu et al. 2020; Wu et al. 2021). In this paper, we provided a method for preparing a Janus membrane which have no selectivity for emulsions that could be used to separate anionic, cationic or nonionic surfactantstabilized emulsions. The growth and fixation of $\mathrm{Mg}(\mathrm{OH})_{2}$ by interfacial ion migration technology modified the surface microstructure of cotton fabrics. Using stearic acid as a coating created the unilateral surface free energy at a suitable lower threshold. Finally, the Janus membrane made by cotton fabric with special wettability was obtained. More importantly, the membrane has no selectivity for the type of surfactants, which means that it could be successfully applied to separating an emulsion stabilized by anionic, cationic or nonionic surfactant.

\section{Experimental}

\subsection{Materials}

Plain woven cotton fabrics (CFs) $\left(110 \mathrm{~g} \mathrm{~m}^{-2}, 52\right.$ warps per $\mathrm{cm}$ and 28 wefts per $\mathrm{cm}$ ) were obtained from the market of China and desized in the $10 \mathrm{~g} / \mathrm{L}$ sodium hydroxide $(\mathrm{NaOH})$ solution at $100^{\circ} \mathrm{C}$ for $60 \mathrm{~min}$. $\mathrm{NaOH}$, urea, stearic acid, reactive turquoise blue, sodium dodecyl sulfate (SDS), cetyltrimethy lammonium bromide (CTAB) and Tween-60 were purchased from China National Medicines Corporation Ltd. All chemicals and reagents were analytical grade had not been further purified.

\subsection{Growth and immobilization of $\mathrm{Mg}(\mathrm{OH})_{2}$}

$7.0 \mathrm{~g}$ of $\mathrm{NaOH}$ was dissolved in $100 \mathrm{~mL}$ of mixed solution with $12.0 \mathrm{~g}$ of urea which was pre-cooled down to $-12^{\circ} \mathrm{C}$. The cotton fabrics $(15 \mathrm{~cm} \times 15 \mathrm{~cm})$ were dipped in the above mixed solution under the condition of $-12^{\circ} \mathrm{C}$ for $2 \mathrm{~h}$. The treated fabrics were squeezed by the padding mangle (MU3C5T, China) with $80 \%$ liquid retention and immediately immersed in $50 \mathrm{wt} \% \mathrm{MgCl}_{2} \cdot 6 \mathrm{H}_{2} \mathrm{O}$ aqueous solution for $10 \mathrm{~s}$ in order to facilitate the magnesium ions to enter the fabric. Then they were transferred into steam machine at $120^{\circ} \mathrm{C}$ for $10 \mathrm{~min}$. The obtained samples were washed with distilled water repeatedly, followed by drying at $40^{\circ} \mathrm{C} . \mathrm{Mg}(\mathrm{OH})_{2}$ was grown in situ and fixed on surface of the cotton fabric.

\subsection{Unilateral hydrophobic treatment of CF}

$0.3 \mathrm{~g}$ of stearic acid was added to $100 \mathrm{~mL}$ ethanol solution with stirring for $5 \mathrm{~min}$. The mixing solution was applied through spray-coating onto the one side of the cotton fabrics with a spray bottle until the surface was covered evenly. A hydrophobic behavior was achieved after drying at $90^{\circ} \mathrm{C}$ for $10 \mathrm{~min}$. The 
above processes were repeated three times and the Janus membrane made by cotton fabric (J-MH@CF) with special wettability was obtained.

\subsection{Characterizations}

The surface morphologies of raw and treated CFs were observed by scanning electron microscopy (SEM, Phenom Pro, Netherlands) with $10 \mathrm{kV}$ accelerating voltage. The crystal structure of CFs was characterized by $X$-ray diffraction (XRD, TD-3500, China) with the monochromatized $\mathrm{Cu} \cdot \mathrm{Ka}_{1}$ radiation $(\lambda=0.154 \mathrm{~nm})$ at $30 \mathrm{kV}$ and $20 \mathrm{~mA}$ over the $2 \theta$ range of $5^{\circ}-70^{\circ}$ in steps of $0.02^{\circ}$. The FTIR spectra were measured by Fourier transform infrared spectroscopy (Nicolet iS10, USA) with wave number range from $4000 \mathrm{~cm}^{-1}$ to $400 \mathrm{~cm}^{-1}$. The mass fraction was recorded by using thermogravimetric analysis (NETZSCH TG 209F3, Germany) under mimetic air atmosphere where the flow rate of Nitrogen and Oxygen was respectively set

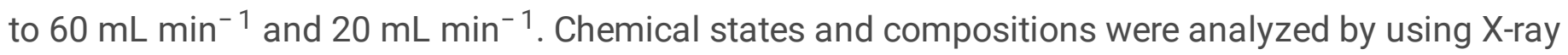
photoelectron spectroscopy (XPS, Thermo Fisher Scientific, USA) equipped with an Al-Ka radiation source

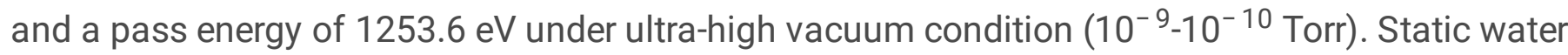
contact angle was collected with a contact angle meter (OCA15EC, Germany) produced by Dataphysics Company in condition that the volume of deionized water droplet was about $3 \mu \mathrm{L}$. Reliable sample angles were measured by averaging the measurements at five different locations on each sample surface.

\subsection{Directional water transmission test}

The J-MH@CF membrane was placed in the air-air interface. When the reverse (hydrophilic) side of JMH@CF was facing up, the water droplets (dyed blue by reactive turquoise blue) spread quickly and did not penetrate down the hydrophobic side (water droplets are blocked). However, when the membrane was reversed, the water droplets permeated spontaneously from the hydrophobic to the hydrophilic side.

To expound insight into the mechanism of the directional movement of water, hypothetical schematic illustrations provided in Fig. 3a. The force analyses of one water droplet permeating a capillary pore of the J-MH@CF membrane from the hydrophobic side to hydrophilic side was described. Synergy of Laplace force $\left(P_{L}\right)$ and hydraulic pressure $\left(P_{H}\right)$ were the main driving force. According to Young-Laplace equation (Eq. (1)), which $P_{L}$ was calculated from, the mechanism of water droplet penetration could be more easily understood (Liu and Lange 2006; Zhou et al. 2013).

$$
\mathrm{P}_{\mathrm{L}}=\frac{2 \gamma \cos \theta}{\mathrm{R}}
$$

where $y$ was the surface tension of liquid, $\theta$ was the contact angle between the capillary wall and water drop, $r$ represented the radius of the liquid "hump".

\subsection{Emulsions separation and recyclability test}

The as-prepared J-MH@CF was flattened between the sand core funnel device (YAMAl, $250 \mathrm{~mL}$, China) so that surfactant-stabilized oil-in-water emulsions were separated under gravity drive. Before implementing the emulsions separation, the J-MH@CF membranes were pre-wetted with distilled water. 
Separation performance of the membrane to emulsions stabilized by surfactants with different ionic type were evaluated in sequence. Anionic surfactant (SDS), cationic surfactant (cetyltrimethy lammonium bromide, CTAB) and nonionic surfactant (Tween-60) were selected as emulsifiers. 1,2-dichloroethane $\left(1.26 \mathrm{~g} \mathrm{~cm}^{-3}\right.$, weight oil), isooctane $\left(0.69 \mathrm{~g} \mathrm{~cm}^{-3}\right.$, light oil) and xylene $\left(0.86 \mathrm{~g} \mathrm{~cm}^{-3}\right.$, light oil) were selected as oil phase. Oil was dyed by Sudan III to differentiate from water.

Oil and water were mixed at a volume ratio of $1: 100$ and the amount of surfactant was $0.1 \mathrm{~g} / \mathrm{L}$. The mixture was treated by high speed homogenizer (XFK FSH-2A, China) at a speed of $13000 \mathrm{rpm}$ for 30s. The surfactant-stabilized oil-in-water emulsion was obtained, and it could be stable for at least 6 hours without delamination.

The gravity-driven separation efficiency and flux were calculated according to the following Eqs. (2) and (3), respectively.

Separation Efficiency $=\frac{V_{P}}{V_{0}} \times 100 \%$

where $V_{0}$ was the initial volume of the water in the mixture before separation and $V_{P}$ was the volume of collected water after separation. The water adhered to the glass instrument and membrane could be precluded.

$\operatorname{Flux}\left(\mathrm{L} / \mathrm{m}^{2} \mathrm{~h}\right)=\frac{V}{S t}$

where $V(L)$ was the volume of filtrates, $S\left(\mathrm{~m}^{2}\right.$, the effective filtration area was $\left.11.9 \mathrm{~cm}^{2}\right)$ was the valid separation area of membrane, $t(h)$ was the separation time.

\section{Results And Discussion}

\subsection{Preparation of J-MH@CF membrane}

The preparation mechanism of J-MH@CF, which combined interfacial ion migration technology and unilateral spraying treatment was illustrated in Scheme $1 \mathrm{a}$. At $-12^{\circ} \mathrm{C}$, the fabric (Scheme $1 \mathrm{c}-\mathrm{Z}$ ) was treated with $7 \mathrm{wt} \% \mathrm{NaOH} / 12 \mathrm{wt} \%$ urea aqueous solution for two hours. $\mathrm{NaOH}$ destroyed the intramolecular and intermolecular hydrogen bonds of cellulose, causing cellulose to swell and even partially dissolve (Scheme 1c- $ه$ ) (Cai and Zhang 2005; Fan et al. 2018). At the same time, hydroxide ions, sodium ions, and alkali cellulose macromolecules constituted internal ionic system of cotton fibers, also called as an intramembrane system (Scheme 1c- $\mathrm{Z}$ ). Alkali cellulose macromolecules were immobilized within the membrane, while hydroxide ions and sodium ions were free to pass through the membrane. When treated cotton fabrics carrying $\mathrm{OH}^{-}$were immersed in $\mathrm{MgCl}_{2} \cdot 6 \mathrm{H}_{2} \mathrm{O}$ aqueous solution, the solution outside the fibers contained magnesium ions $\left(\mathrm{Mg}^{2+}\right)$ and chlorine ions, also considered as an extra-membrane 
system. There was concentration gradient of mobile ions between inside and outside the membrane. Therefore, $\mathrm{Mg}^{2+}$ tended to migrate to the intra-membrane system and $\mathrm{OH}^{-}$was inclined to move to the extra-membrane system. $\mathrm{Mg}^{2+}$ reacted with the $\mathrm{OH}^{-}$immediately to form the crystallization of magnesium hydroxide at the interface of the membrane (surface of cotton fibers). As shown in SEM images, the raw CF surface was smooth, whereas the fibers of J-MH@CF were fully clad by $\mathrm{Mg}(\mathrm{OH})_{2}$ with cross-connected micro-nanostructure. Stearic acid possessed relatively low surface energy and could impart favorable hydrophobicity to substrates. After spraying stearic acid solution, the - $\mathrm{COOH}$ of stearic acid reacted with $\mathrm{Mg}(\mathrm{OH})_{2}$, and formed the stearate to modified the surface of $\mathrm{Mg}(\mathrm{OH})_{2}$ (Haworth et al. 2000; Zhang et al. 2007). The mimetic core $\left(\mathrm{Mg}(\mathrm{OH})_{2}\right)$ /shell (stearate) structure was produced spontaneously. This structure as a coating layer created the unilateral surface free energy at a suitable lower threshold.

Figure 1a shows the FTIR spectra obtained for raw CF and J-MH@CF. Absorption bands at $2968 \mathrm{~cm}^{-1}$ and $2918 \mathrm{~cm}^{-1}$ were belonging to the asymmetrical stretching vibration of $\mathrm{C}-\mathrm{H}$ bond in $-\mathrm{CH}_{3}$ and $-\mathrm{CH}_{2}$ group, respectively. Absorption bands at $2848 \mathrm{~cm}^{-1}$ was due to the symmetrical stretching vibration of C$\mathrm{H}$ bond in $-\mathrm{CH}_{2}$ group. The peak at $1540 \mathrm{~cm}^{-1}$ and $1457 \mathrm{~cm}^{-1} 1$ was caused by the functional group of carbonyl group $(C=0)$ (Wen et al. 2018; Khattab et al. 2020; sharif et al. 2020). The intensity and position of the peak show that the stearic acid was successfully applied to the fabric surface. In the XRD pattern (Fig. 1b), both samples show three peaks centered around $14.94^{\circ}, 16.69^{\circ}$, and $22.79^{\circ}$, which were in accordance with the typical (1-1 0), (1 10$)$ and (2 00 ) peaks of natural cellulose fibers (French 2014). It was clear that the characteristic peaks indicated $\mathrm{Mg}(\mathrm{OH})_{2}$ phase $\left(2 \theta=19.22^{\circ}, 38.30^{\circ}, 50.78^{\circ}\right.$ and $\left.58.96^{\circ}\right)$, which were attributed to their corresponding indices $\left(\begin{array}{lll}0 & 0 & 1\end{array}\right),\left(\begin{array}{lll}1 & 0 & 1\end{array}\right),\left(\begin{array}{lll}1 & 0 & 2\end{array}\right)$ and $\left(\begin{array}{lll}1 & 1 & 0\end{array}\right)$, respectively. The synthesis of $\mathrm{Mg}(\mathrm{OH})_{2}$ was further confirmed by XPS. From the wide scan spectrum (Fig. 1d), both CF and J-MH@CF had two main peaks of C1s and 01s. J-MH@CF has core level peaks of Mg 2p, Mg 2s and Mg 1s at $49.62 \mathrm{eV}, 88.08 \mathrm{eV}$ and $1303.17 \mathrm{eV}$, while $\mathrm{Mg}$ (KLL) peak appeared at about $306.08 \mathrm{eV}$ (Keikhaei and Ichimura 2019). As shown in Fig. $1 \mathrm{e}$ and $1 \mathrm{f}, \mathrm{Mg} 2 \mathrm{p}$ peak at $49.26 \mathrm{eV}$ and the $01 \mathrm{~s}$ peak at $531.02 \mathrm{eV}$ in XPS spectra was related to $\mathrm{Mg}(\mathrm{OH})_{2}$ (Ardizzone et al. 1997; Zhu et al. 2011). The residual mass of J$\mathrm{MH} @ \mathrm{CF}$ at $700^{\circ} \mathrm{C}$ further verified the existence of $\mathrm{Mg}(\mathrm{OH})_{2}$ on cotton fabrics and implied the magnesium hydroxide content of about $4 \%$ (Fig. 1C).

\subsection{Wettability and water contact angle}

The obverse(hydrophobic) side had hydrophobic abilities with high WCA of about $141.1^{\circ}$ and the reverse(hydrophilic) side had hydrophilicity abilities with an WCA of about $41.2^{\circ}$. After dripping water onto the obverse and reverse sides of J-MH@CF membrane, the change of contact angle was recorded separately in Fig. 2a. Figure 2c is a series of digital photographs and shows the wettability of J-MH@CF in the air. This wetting process was also simulated in Fig. 2b. When the water was dropped onto the obverse side, the water droplet could spontaneously penetrate the J-MH@CF from the hydrophobic side to the hydrophilic side. Compared to the opposite side, droplets only moistened the hydrophilic side and spread out. It took about $15.4 \mathrm{~s}$ for water droplet to penetrate the membrane completely. After being 
dropped on the reverse side, water droplet diffused rapidly to the hydrophilic side surface without penetration. The surface of hydrophilic side was wetted. After 2.9 seconds, water droplet completely spread on the hydrophilic side.

\subsection{Evaluation of water-unidirectional permeation movement}

In a typical directional movement of water, water droplets penetrated through the hydrophobic channels of the J-MH@CF membrane and reached the hydrophilic layer. The permeation process was simulated in Fig. 3a. A water droplet dropped onto the hydrophobic side should form a hump (i.e., $P_{L}$ ) on the opposite side of the membrane pores if there is nothing underneath. However, because of the presence of hydrophilic capillary force, the hydrophilic layer underneath would eliminate $\mathrm{P}_{\mathrm{L}}$ once the "hump" contacted the hydrophilic layer (Chen et al. 2019). On the contrary, water droplet spread into a thin film quickly when it dropped onto the hydrophilic side and it will be rejected at the junction of hydrophilic and hydrophobic layers. In order to minimize $\mathrm{P}_{\mathrm{L}}$, the thinner the hydrophobic layer, the better. The combination of thick hydrophilic layer and thin hydrophobic layer could realize the fast directional water transmission. Figure $2 \mathrm{~b}$ provide a set of pictures to show the unidirectional transfer behavior of water in the air/air system. Having shown the working mechanism for air-air system, the directional movement of water of J-

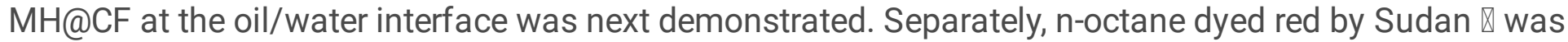
used as a model oil, and water was dyed blue by reactive turquoise blue to distinguish it from the oil. The droplet penetrates easily through the J-MH@CF membrane forming shapes similar to flammulina velutipes (Fig. 2c).

\subsection{Evaluation of the emulsion separation performance}

The high wettability to continuous phase and high hindrance to dispersed phase of the obtained membrane endowed it with an excellent capability for separating surfactant-stabilized oil-in-water emulsions. Hence, a series of separation experiments were carried out to evaluate the performance of JMH@FF membrane. Emulsions stabilized by three different ionic types of surfactants (SDS, CTAB or Tween-60) were used as model emulsions and emulsions were derived from 1,2-dichloroethane (1.26 g $\mathrm{cm}^{-3}$, weight oil), isooctane $\left(0.69 \mathrm{~g} \mathrm{~cm}^{-3}\right.$, light oil) and xylenes $\left(0.86 \mathrm{~g} \mathrm{~cm}^{-3}\right.$, light oil). The anionic surfactant stabilized emulsion (1,2-dichloroethane/SDS/ $\mathrm{H}_{2} \mathrm{O}$ ) separation was evaluated at first.

As shown in Fig. 4a, emulsion was directly injected into a sand core funnel device, and the volume of the emulsion was fixed as $100 \mathrm{~mL}$. What needed to be mentioned was that all the separations were purely gravity driven only which was the same as a driving pressure of $\approx 1 \mathrm{kPa}$. The emulsion was separated rapidly with water almost entirely penetrating the membrane while the oil droplets surrounded by SDS was blocked at the upper beaker. Optical microscopic images (Fig. 4c) of the emulsion and filtrate indicated that both emulsions contained numerous unordered oil droplets, while the filtrate did not. Furthermore, as shown in the digital photographs, all the filtrates were clear and transparent. In the spectrum of SDS, absorption bands at $2971.73 \mathrm{~cm}^{-1}$ and $2868.71 \mathrm{~cm}^{-1}$ were mainly dominated by the symmetric and asymmetric stretching bands of $\mathrm{C}-\mathrm{H}$ of the hydrophobic (tail) groups. Peaks at 1200.15 $\mathrm{cm}^{-1}$ resulted from asymmetric S-0 stretching, whereas the peaks at $1004.29 \mathrm{~cm}^{-1}$ corresponded to 
symmetric S-O stretching (Gao and Chorover 2010; Ramimoghadam et al. 2012; Warsi et al. 2020). Compared with the emulsion, the peaks of SDS $\left(2971.73 \mathrm{~cm}^{-1}, 2868.71 \mathrm{~cm}^{-1}\right)$ were not highlighted in the curve of the filtrate. FTIR pattern (Fig. 4b) of the filtrate was similar to that of distilled water, which showed that the separation effect was satisfactory.

For emulsions derived from different kinds of oil (Fig. 4d), 1,2-dichloroethane/SDS/ $\mathrm{H}_{2} \mathrm{O}$, isooctane/SDS/ $\mathrm{H}_{2} \mathrm{O}$, xylene/SDS/ $\mathrm{H}_{2} \mathrm{O}$, the separation flux was $532 \mathrm{~L} \mathrm{~m}^{-2} \mathrm{~h}^{-1}, 609 \mathrm{~L} \mathrm{~m}^{-2} \mathrm{~h}^{-1}$ and $426 \mathrm{~L}$ $\mathrm{m}^{-2} \mathrm{~h}^{-1}$, respectively. After calculation, separation efficiencies were more than $99.3 \%$ each time and data of detailed flux were also shown in the Fig. $4 \mathrm{~d}$.

\subsection{Separation mechanism}

Separation process was demonstrated in Fig. 5a based on the simulation to further showing the mechanism of the J-MH@CF separation performance. SDS molecule included a 12 carbon saturated alkyl chain(tail) and a negatively charged sulfonate head $\left(-\mathrm{OSO}_{3}{ }^{-}\right)$. After SDS was added to the oil-water mixture, the surfactant coating structure of the oil droplet was gradually assembled, indicating molecular aggregated. Oil droplet surrounded by hydrophobic tails of surfactant tended to form the core, while the hydrophilic heads were exposed outside in contact with the aqueous environment. When the emulsion was separated, water formed a "hump" (i.e., $\mathrm{P}_{\mathrm{L}}$ ) at the hydrophobic side of the membrane pores, and because of the presence of hydrophilic capillary force, the hydrophilic layer underneath would eliminate $\mathrm{P}_{\mathrm{L}}$ once the "hump" contacted the hydrophilic layer (Hou et al. 2019). Water as a continuous phase penetrated the membrane. Oil droplets surrounded by SDS were difficult to aggregate due to their thermodynamic stability. The $\mathrm{Mg}(\mathrm{OH})_{2}$ decorated $\mathrm{CF}$ with an increase in micro/nanostructure and a reduction in the contact area between oil droplets and membrane (Zhang et al. 2013). Unilateral modification of stearic acid further exposed a large number of carbon chains on the surface of micro/nanostructures, resulting in a reduction in surface energy. Therefore, there was a repulsive force between the oil droplets surrounded by SDS and the carbon chains. Under the action of the repulsive force and the stable thermodynamic state of the oil droplets, it was difficult for the oil droplets to demulsify to spread or wet on the membrane. Such a wetting behavior was Cassie-Baxter wetting state, and oil droplets surrounded by SDS as a dispersed phase were rejected by the membrane. As time progressed, the "cream layer" was gradually formed.

In the same way, when CTAB or Tween-60 was added to oil-water mixture, its hydrophilic groups were exposed outside in contact with the aqueous environment and the hydrophobic groups were in contact with the oil, indicating that a stable structure similar to SDS coating structure of the oil droplet was also formed. However, due to the different types of surfactant or oil, there was likely to be a vastly difference in the size and shape of the oil droplets surrounded by surfactants, resulting in differential flux. For emulsions by surfactants with different ionic type, as shown in the Fig. 5b-5d, CTAB/Oil/ $\mathrm{H}_{2} \mathrm{O}$ emulsions and the Tween-60/Oil/ $\mathrm{H}_{2} \mathrm{O}$ emulsions could also be successfully separated with high separation efficiency, indicating that the J-MH@CF membrane could be used to separate oily wastewater containing unspecified surfactants. 


\subsection{Cycle performance}

The "cream layer" gradually formed on the surface of the membrane in the separation process (Fig. 6a). In order to determine the existence state of surfactant, oil and water, the "cream layer" obtained after the separation of $50 \mathrm{~mL}, 100 \mathrm{~mL}$ and $150 \mathrm{~mL}$ emulsion were observed by optical microscope respectively. From the optical microscope pictures (Fig. 6b), it could be seen that the accumulation of emulsion droplets became more and more obvious with the increase of emulsion content, which showed that the "cream layer" was composed of undemulsified emulsion droplets. In addition, the "cream layer" contained peaks $\left(2971.73 \mathrm{~cm}^{-1}, 2868.71 \mathrm{~cm}^{-1}\right)$ that represent existence of SDS (Fig. 6c). After rinsing with distilled water, the "cream layer" was easily washed off and the membrane was not contaminated by oil droplets (Fig. 6f). There was no obvious decrease in liquid flux and separation efficiency after 10 cycles, which indicated robust reusability (Fig. 6e). Oil droplets accumulated gradually driven by gravity on the membrane surface to form a "cream layer", which facilitated to improve the separation efficiency to a certain extent. However, with the increase of the thickness, the "cream layer" also gradually covered the surface pores and reduced the effective separation area of the membranes, resulting in a gradual reduction of separation flux. For surfactant-stabilized emulsions by SDS, after each $5 \mathrm{~mL}$ of filtrate was collected, the flux was calculated (Fig. $6 \mathrm{~d}$ ). As the separation process went on, flux gradually decreased from $1403 \mathrm{~m}^{-2} \mathrm{~h}^{-1}$ to about $412 \mathrm{~L} \mathrm{~m}^{-2} \mathrm{~h}^{-1}$.

\section{Conclusion}

In summary, J-MH@CF membranes involving asymmetric wettability were prepared by interfacial ion migration technology and unilateral spraying treatment, which involved surface roughness structure increased by immobilization of $\mathrm{Mg}(\mathrm{OH})_{2}$ and relatively low surface energy modified by stearic acid. When surfactant-stabilized oil-in-water emulsions was separated, the resultant J-MH@CF membranes presented directional transport of water droplets and selective rejection of oil droplets. The J-MH@CF membrane exhibited the permeate flux of emulsions in the sequence of isooctane/SDS/ $\mathrm{H}_{2} \mathrm{O}\left(609 \mathrm{~L} \mathrm{~m}^{-2} \mathrm{~h}^{-1}\right)>1$,2dichloroethane/SDS/ $\mathrm{H}_{2} \mathrm{O}\left(532 \mathrm{~L} \mathrm{~m}^{-2} \mathrm{~h}^{-1}\right)>x y$ lene/SDS/ $\mathrm{H}_{2} \mathrm{O}\left(426 \mathrm{~L} \mathrm{~m}^{-2} \mathrm{~h}^{-1}\right)$ with separation efficiency of 99.6\%, 99.5\% and 99.6\%, respectively. Importantly, J-MH@CF membranes were effective for separating oil-in-water emulsions stabilized by SDS, CTAB or Tween-60, with a high separation flux and efficiency driven only by gravity. Furthermore, the "cream layer" formed by the settling of oil droplets during the separation process could also be washed off and the membranes showed excellent stability and reusability in a 10-cycle test. These results proved that J-MH@CF membranes was an expected separation material and showed a vast range of application prospects in separation of oily wastewater containing unspecified surfactants.

\section{Declarations}

\section{Acknowledgements}


Pu Yang and Ruimin Hu contributed equally to this work. This work was financially supported by Government-Sponsored Visiting Scholar Research Program (201908505032, 202006995001) and Southwest University Undergraduate Innovation and Entrepreneurship Training Program (X202010635408).

\section{References}

An YP, Yang J, Yang HC, et al (2018) Janus Membranes with Charged Carbon Nanotube Coatings for Deemulsification and Separation of Oil-in-Water Emulsions. ACS Appl Mater Interfaces 10:9832-9840. https://doi.org/10.1021/acsami.7b19700

Ardizzone S, Bianchi CL, Fadoni M, Vercelli B (1997) Magnesium salts and oxide: an XPS overview. Appl Surf Sci 119:253-259. https://doi.org/10.1016/s0169-4332(97)00180-3

Cai J, Zhang L (2005) Rapid dissolution of cellulose in $\mathrm{LiOH} /$ urea and $\mathrm{NaOH} /$ urea aqueous solutions. Macromol Biosci 5:539-548. https://doi.org/10.1002/mabi.200400222

Chen C, Weng D, Mahmood A, et al (2019) Separation Mechanism and Construction of Surfaces with Special Wettability for Oil/Water Separation. ACS Appl Mater Interfaces 11:11006-11027. https://doi.org/10.1021/acsami.9b01293

Dai B, Li K, Shi L, et al (2019) Bioinspired Janus Textile with Conical Micropores for Human Body Moisture and Thermal Management. Adv Mater 31:1-7. https://doi.org/10.1002/adma.201904113

Fan T, Qian Q, Hou Z, et al (2018) Preparation of smart and reversible wettability cellulose fabrics for oil/water separation using a facile and economical method. Carbohydr Polym 200:63-71. https://doi.org/10.1016/j.carbpol.2018.07.040

French AD (2014) Idealized powder diffraction patterns for cellulose polymorphs. Cellulose 21:885-896. https://doi.org/10.1007/s10570-013-0030-4

Gao X, Chorover J (2010) Adsorption of sodium dodecyl sulfate (SDS) at ZnSe and a-Fe2O3 surfaces: Combining infrared spectroscopy and batch uptake studies. J Colloid Interface Sci 348:167-176. https://doi.org/10.1016/j.jcis.2010.04.011

Gao Y, Wang J, Xia W, et al (2018) Reusable Hydrophilic-Superhydrophobic Patterned Weft Backed Woven Fabric for High-Efficiency Water-Harvesting Application. ACS Sustain Chem Eng 6:7216-7220. https://doi.org/10.1021/acssuschemeng.8b01387

Ge J, Zong D, Jin Q, et al (2018) Biomimetic and Superwettable Nanofibrous Skins for Highly Efficient Separation of Oil-in-Water Emulsions. Adv Funct Mater 28:1-10. https://doi.org/10.1002/adfm.201705051 
Gupta RK, Dunderdale GJ, England MW, Hozumi A (2017) Oil/water separation techniques: a review of recent progresses and future directions. J Mater Chem A 5:16025-16058.

https://doi.org/10.1039/c7ta02070h

Haworth B, Raymond CL, Sutherland I (2000) Polyethylene compounds containing mineral fillers modified by acid coatings. 1: Characterization and processing. Polym Eng Sci 40:1953-1968.

https://doi.org/10.1002/pen.11327

Hou L, Wang N, Man X, et al (2019) Interpenetrating janus membrane for high rectification ratio liquid unidirectional penetration. ACS Nano 13:4124-4132. https://doi.org/10.1021/acsnano.8b08753

Keikhaei M, Ichimura M (2019) Fabrication of $\mathrm{Mg}(\mathrm{OH}) 2$ thin films by electrochemical deposition with $\mathrm{Cu}$ catalyst. Thin Solid Films 681:41-46. https://doi.org/10.1016/j.tsf.2019.04.046

Khattab TA, Mohamed AL, Hassabo AG (2020) Development of durable superhydrophobic cotton fabrics coated with silicone/stearic acid using different cross-linkers. Mater Chem Phys 249:122981.

https://doi.org/10.1016/j.matchemphys.2020.122981

Li D, Lin J, An Z, et al (2020) Enhancing hydrophilicity and comprehensive antifouling properties of microfiltration membrane by novel hyperbranched poly(N-acryoyl morpholine) coating for oil-in-water emulsion separation. React Funct Polym 156:104735.

https://doi.org/10.1016/j.reactfunctpolym.2020.104735

Li H, Cao M, Ma X, et al (2016) "Plug-and-Go"-Type Liquid Diode: Integrated Mesh with Janus Superwetting Properties. Adv Mater Interfaces 3:. https://doi.org/10.1002/admi.201600276

Lin X, Heo J, Choi M, Hong J (2019) Simply realizing durable dual Janus superwettable membranes integrating underwater low-oil-adhesive with super-water-repellent surfaces for controlled oil-water permeation. J Memb Sci 580:248-255. https://doi.org/10.1016/j.memsci.2019.03.038

Liu B, Lange FF (2006) Pressure induced transition between superhydrophobic states: Configuration diagrams and effect of surface feature size. J Colloid Interface Sci 298:899-909. https://doi.org/10.1016/j.jcis.2006.01.025

Liu HF, Wang Z, Sun CJ (2020) Scalable and rapid preparation of Janus fabric by trans-printing method for efficient oil/water emulsion separation. J Dispers Sci Technol 41:1236-1245. https://doi.org/10.1080/01932691.2019.1615934

Liu L, Pan Y, Bhushan B, Zhao X (2019) Mechanochemical robust, magnetic-driven, superhydrophobic 3D porous materials for contaminated oil recovery. J Colloid Interface Sci 538:25-33. https://doi.org/10.1016/j.jcis.2018.11.066

Liu Y, Su Y, Cao J, et al (2017a) Antifouling, high-flux oil/water separation carbon nanotube membranes by polymer-mediated surface charging and hydrophilization. J Memb Sci 542:254-263. 
https://doi.org/10.1016/j.memsci.2017.08.018

Liu YQ, Han DD, Jiao ZZ, et al (2017b) Laser-structured Janus wire mesh for efficient oil-water separation. Nanoscale 9:17933-17938. https://doi.org/10.1039/c7nr06110b

Manna U, Lynn DM (2015) Synthetic Surfaces with Robust and Tunable Underwater Superoleophobicity. Adv Funct Mater 25:1672-1681. https://doi.org/10.1002/adfm.201403735

McClements DJ, Jafari SM (2018) Improving emulsion formation, stability and performance using mixed emulsifiers: A review. Adv Colloid Interface Sci 251:55-79. https://doi.org/10.1016/j.cis.2017.12.001

Ostertag F, Weiss J, McClements DJ (2012) Low-energy formation of edible nanoemulsions: Factors influencing droplet size produced by emulsion phase inversion. J Colloid Interface Sci 388:95-102. https://doi.org/10.1016/j.jcis.2012.07.089

Panwar K, Jassal M, Agrawal AK (2018) TiO2-SiO2 Janus particles for photocatalytic self-cleaning of cotton fabric. Cellulose 25:2711-2720. https://doi.org/10.1007/s10570-018-1698-2

Ramimoghadam D, Hussein MZ Bin, Taufiq-Yap YH (2012) The effect of sodium dodecyl sulfate (SDS) and cetyltrimethylammonium bromide (CTAB) on the properties of $\mathrm{ZnO}$ synthesized by hydrothermal method. Int J Mol Sci 13:13275-13293. https://doi.org/10.3390/ijms131013275

sharif R, Mohsin M, Ramzan N, et al (2020) Synthesis and Application of Fluorine-free Environmentfriendly Stearic Acid-based Oil and Water Repellent for Cotton Fabric. J Nat Fibers 00:1-16. https://doi.org/10.1080/15440478.2020.1787918

Wang Y, Liang X, Zhu H, et al (2020) Reversible Water Transportation Diode: Temperature-Adaptive Smart Janus Textile for Moisture/Thermal Management. Adv Funct Mater 30:1-9.

https://doi.org/10.1002/adfm.201907851

Wang Z, Lehtinen M, Liu G (2017a) Universal Janus Filters for the Rapid Separation of Oil from Emulsions Stabilized by lonic or Nonionic Surfactants. Angew Chemie 129:13072-13077. https://doi.org/10.1002/ange.201706158

Wang Z, Wang Y, Liu G (2016) Rapid and Efficient Separation of Oil from Oil-in-Water Emulsions Using a Janus Cotton Fabric. Angew Chemie-International Ed 55:1291-1294.

https://doi.org/10.1002/anie.201507451

Wang Z, Yang X, Cheng Z, et al (2017b) Simply realizing "water diode" Janus membranes for multifunctional smart applications. Mater Horizons 4:701-708. https://doi.org/10.1039/c7mh00216e

Warsi F, Islam MR, Alam MS, Ali M (2020) Exploring the effect of hydrophobic ionic liquid on aggregation, micropolarity and microviscosity properties of aqueous SDS solutions. J Mol Liq 310:113132. https://doi.org/10.1016/j.molliq.2020.113132 
Wen R, Zhang W, Lv Z, et al (2018) A novel composite Phase change material of Stearic Acid/Carbonized sunflower straw for thermal energy storage. Mater Lett 215:42-45.

https://doi.org/10.1016/j.matlet.2017.12.008

Woo S, Cho H, Park J, et al (2020) A novel approach to designing a biomimetic wettable patterned surface for highly efficient and continuous surfactant-free oil emulsion separation. Sep Purif Technol 248:116864. https://doi.org/10.1016/j.seppur.2020.116864

Wu Z, Zhang T, Zhang H, et al (2021) One-pot fabrication of hydrophilic-oleophobic cellulose nanofibersilane composite aerogels for selectively absorbing water from oil-water mixtures. Cellulose 0123456789: https://doi.org/10.1007/s10570-020-03610-y

Xu L, Liu Y, Yuan X, et al (2020) One-pot preparation of robust, ultraviolet-proof superhydrophobic cotton fabrics for self-cleaning and oil/water separation. Cellulose 27:9005-9026.

https://doi.org/10.1007/s10570-020-03369-2

Yao C, Luo M, Wang H, et al (2019) Asymmetric wetting Janus fabrics with double-woven structure for oil/water separation. J Mater Sci 54:5942-5951. https://doi.org/10.1007/s10853-018-03241-6

Zhang F, Zhang W Bin, Shi Z, et al (2013) Nanowire-haired inorganic membranes with superhydrophilicity and underwater ultralow adhesive superoleophobicity for high-efficiency oil/water separation. Adv Mater 25:4192-4198. https://doi.org/10.1002/adma.201301480

Zhang F, Zhang H, Su Z (2007) Surface treatment of magnesium hydroxide to improve its dispersion in organic phase by the ultrasonic technique. Appl Surf Sci 253:7393-7397.

https://doi.org/10.1016/j.apsusc.2007.03.022

Zhang W, Li X, Qu R, et al (2019) Janus membrane decorated: Via a versatile immersion-spray route: Controllable stabilized oil/water emulsion separation satisfying industrial emission and purification criteria. J Mater Chem A 7:4941-4949. https://doi.org/10.1039/c8ta11452h

Zhou H, Wang H, Niu H, Lin T (2013) Superphobicity/philicity Janus Fabrics with Switchable, Spontaneous, Directional Transport Ability to Water and Oil Fluids. Sci Rep 3:2964. https://doi.org/10.1038/srep02964

Zhu Y, Wu G, Zhang YH, Zhao Q (2011) Growth and characterization of $\mathrm{Mg}(\mathrm{OH}) 2$ film on magnesium alloy AZ31. Appl Surf Sci 257:6129-6137. https://doi.org/10.1016/j.apsusc.2011.02.017

Zuo J-H, Gu Y-H, Wei C, et al (2020) Janus polyvinylidene fluoride membranes fabricated with thermally induced phase separation and spray-coating technique for the separations of both W/O and O/W emulsions. J Memb Sci 595:117475. https://doi.org/10.1016/j.memsci.2019.117475

\section{Figures}



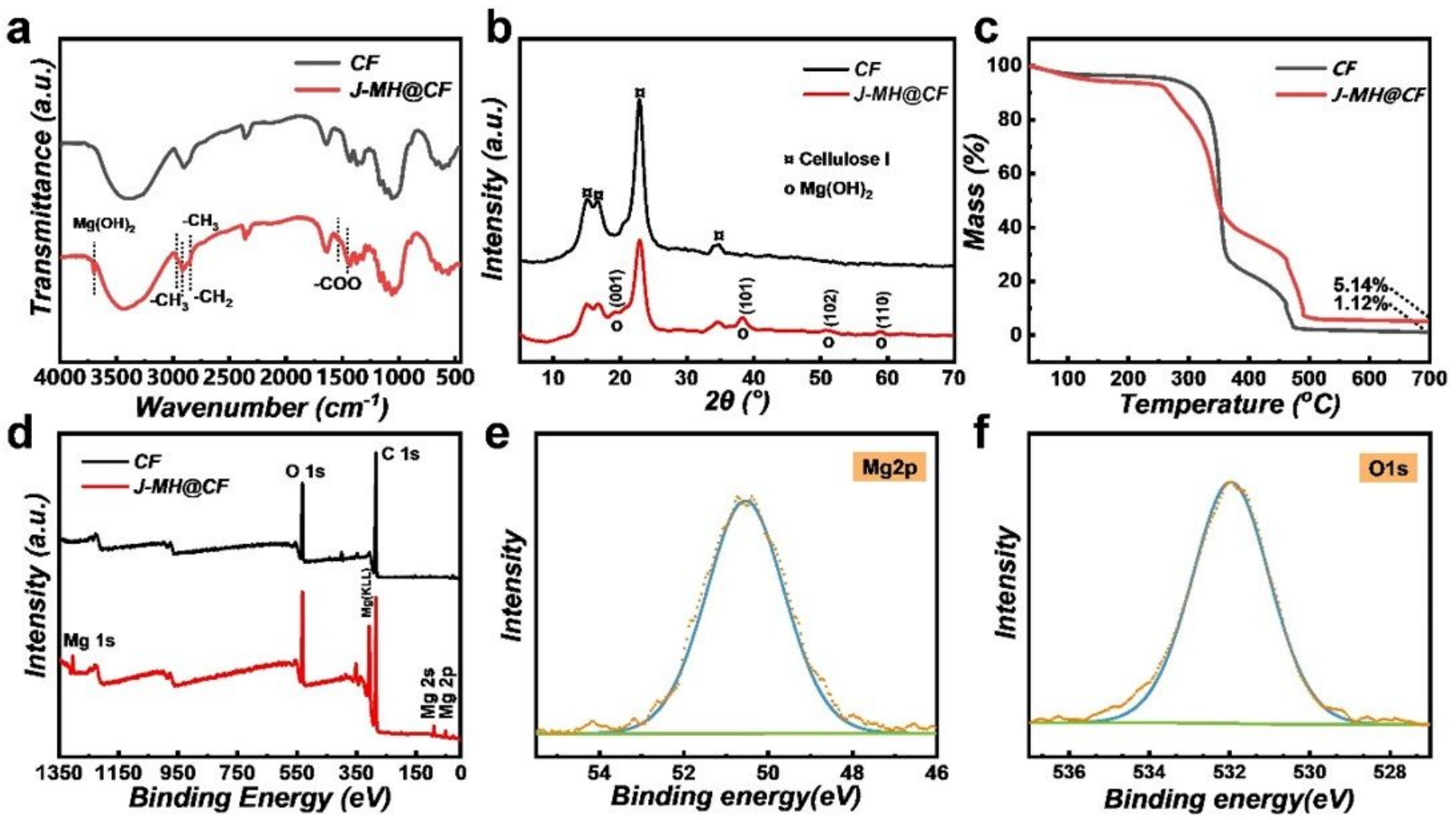

Figure 1

(a) FTIR (b)XRD (c) TG (d)XPS survey spectra of raw CF and J-MH@CF membrane, (e) 0 1s (f) Mg 2p of J-MH@CF membrane. 


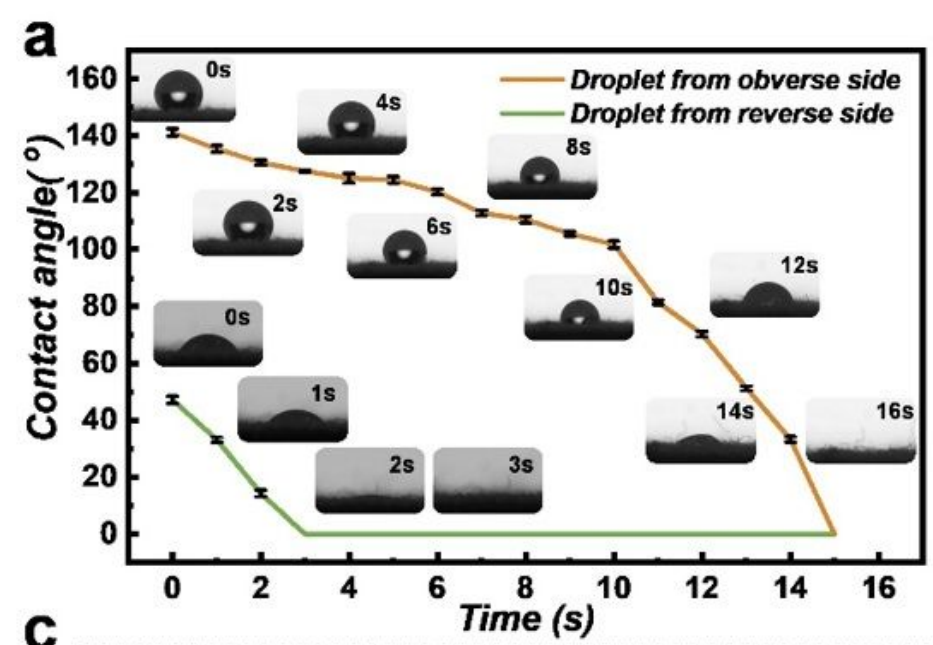

b

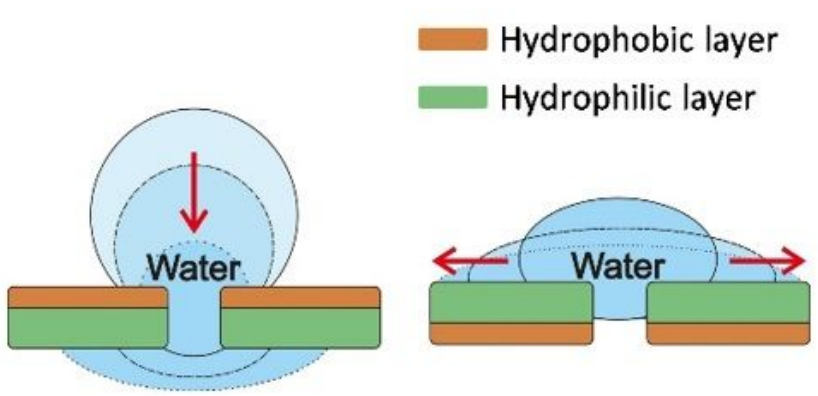

Droplet from obverse side Droplet from reverse side

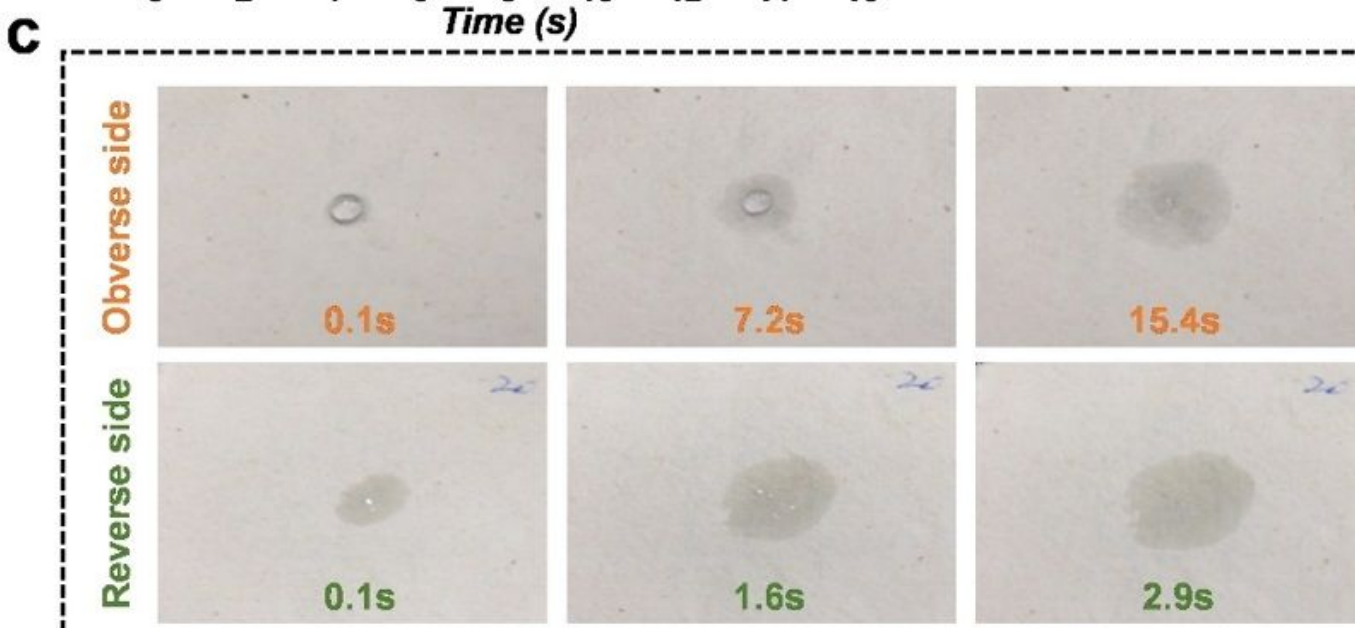

Time

Opposite side

Figure 2

(a) Water contact angle changes during the dropping of water onto the obverse and reverse side of the JMH@CF membrane. (b) Schematic diagram of the mechanism of wetting process. (c) A series of photographs with difference in wetting when dripping on the obverse and reverse side of the J-MH@CF membrane. 
a

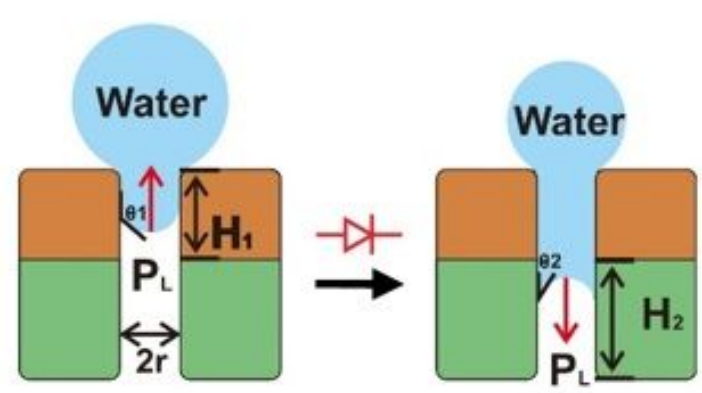

Hydrophobic layer Hydrophilic layer

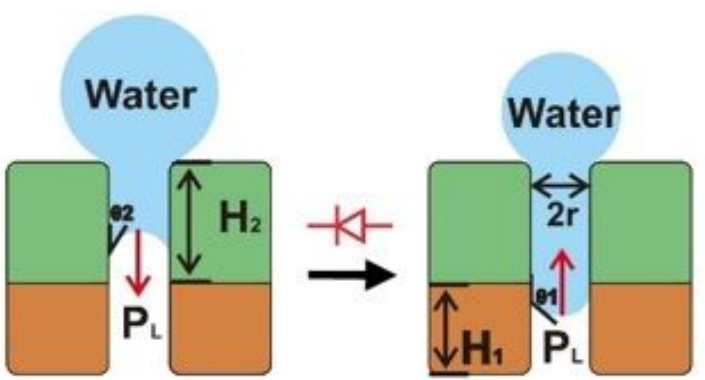

Hydrophobic layer Hydrophilic layer

b
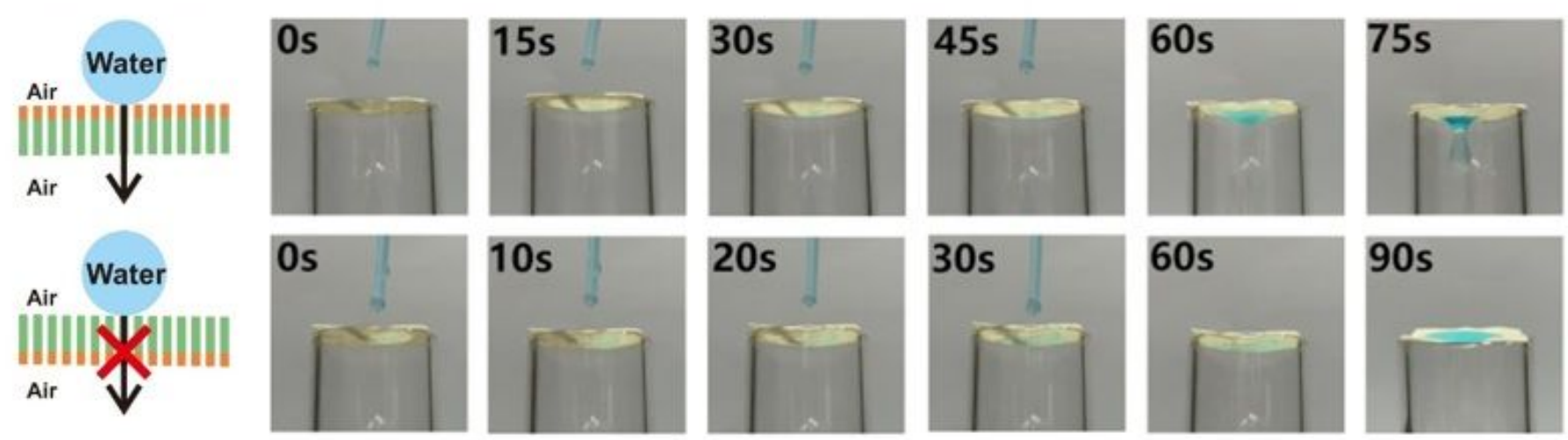

C
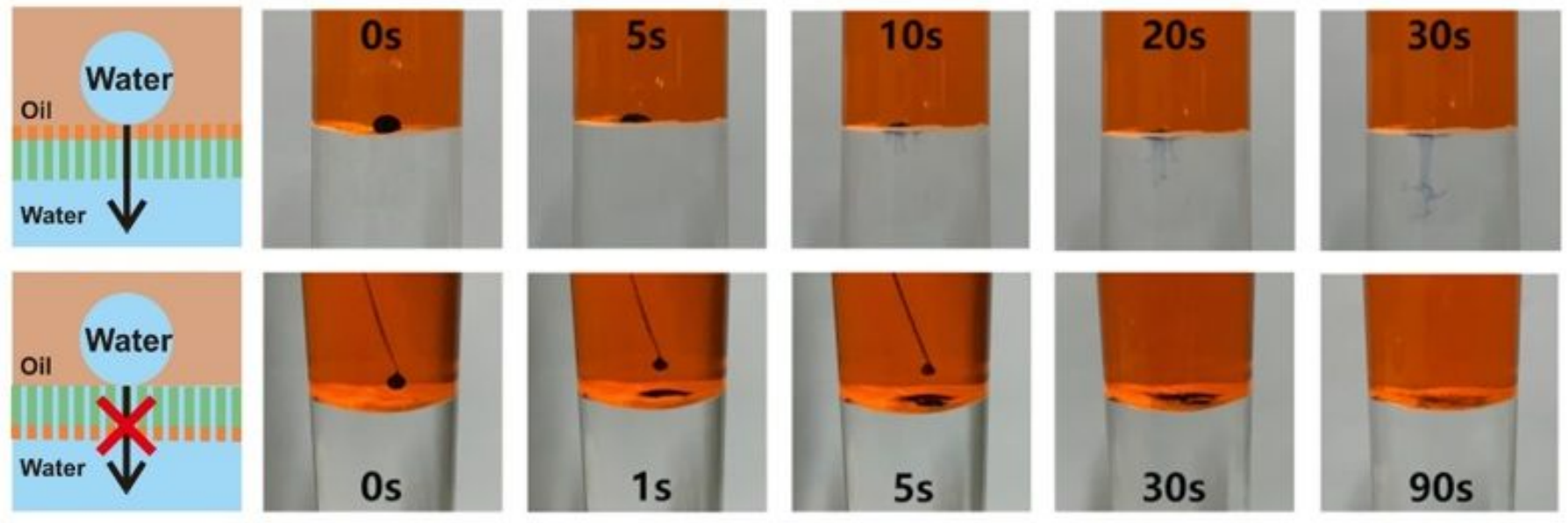

\section{Figure 3}

(a) Schematic diagram of the mechanism of the water-unidirectional permeation movement. The schematic and photographs of water-unidirectional behaviors of J-MH@CF membrane with different fixed modes at (b) air/air system and (c) oil/water system. 

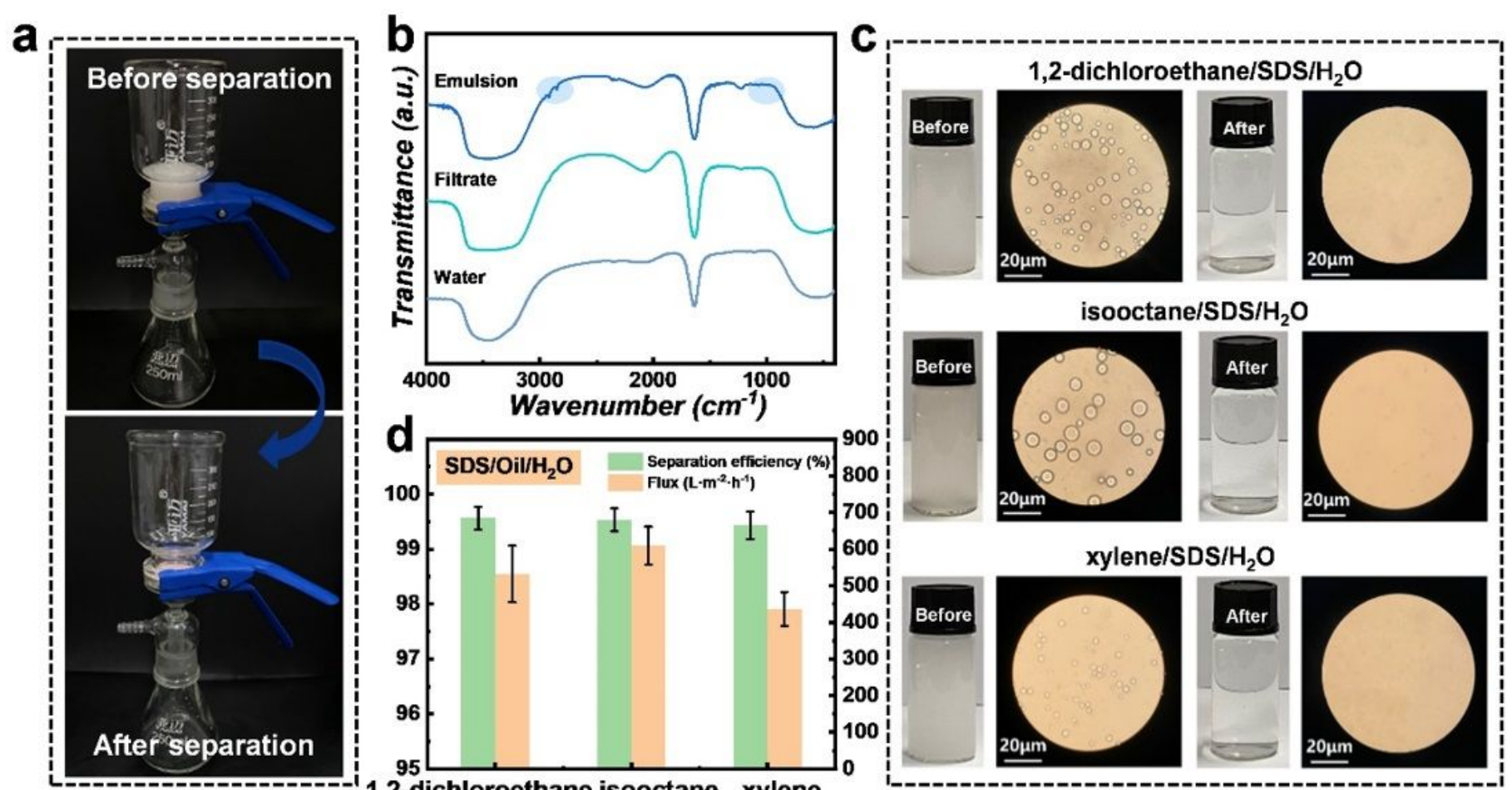

1,2-dichloroethane isooctane xylene

\section{Figure 4}

(a) Photographs of gravity-driven separation processes, (b) FTIR spectra of emulsion, filtrate and water.

(c) Photographs and optical microscopic images of the surfactant-stabilized emulsions before and after separation, (d) Separation efficiency and permeation flux for different emulsions stabilized by SDS under the driving of gravity. 


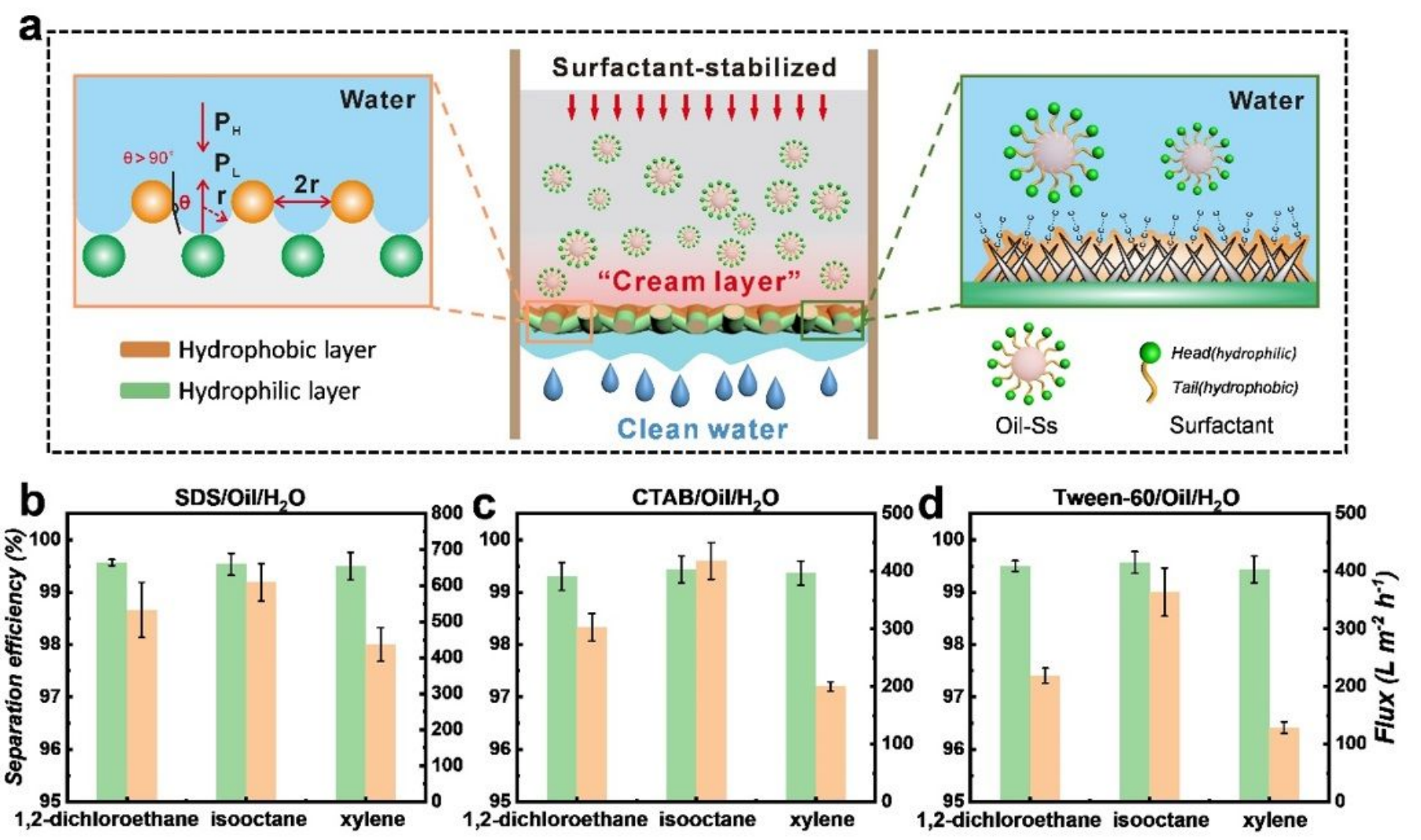

Figure 5

(a) Schematic diagram of the mechanism of the emulsions separation. (b-d) Separation efficiency and flux of J-MH@CF for SDS/Oil/H2O emulsion, CTAB/Oil/H2O emulsion and Tween-60/Oil/H2O emulsion. 

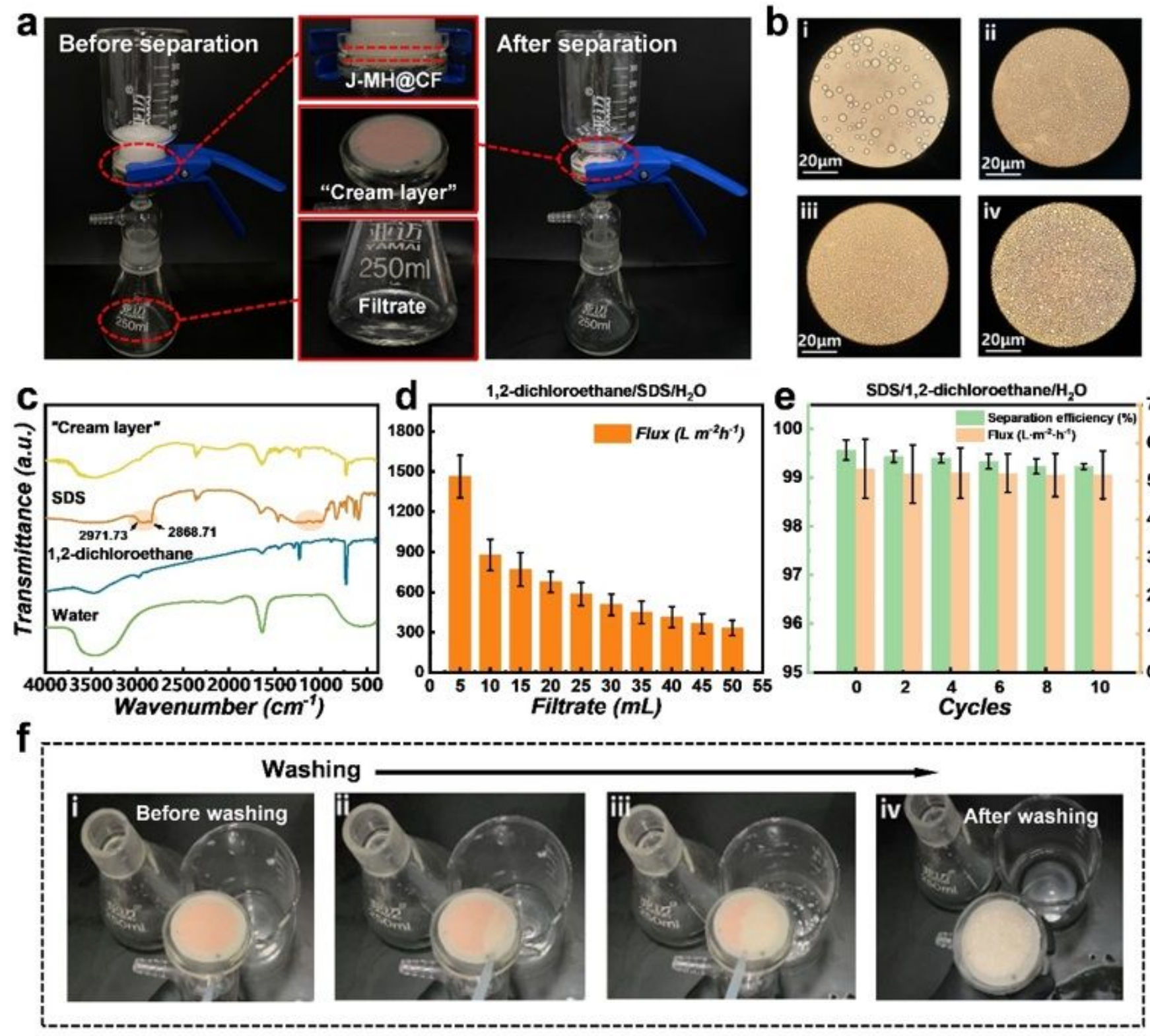

\section{Figure 6}

(a) Photographs of the emulsion before and after separation and of the "cream layer" resulting from the separation. (b) Optical microscopic images of the emulsion ( $b-\rrbracket)$ and "cream layer" obtained after the

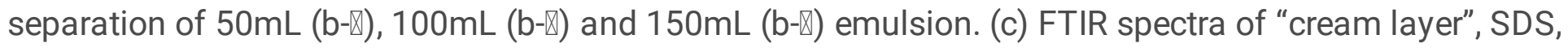
oil and water. (d) Flux calculated after every $5 \mathrm{~mL}$ of filtrate collected during separation. (e) The recyclability of J-MH@CF membrane for surfactant-stabilized emulsions separation. (f) Photographs of the process of rinsing off the "cream layer" with water.

\section{Supplementary Files}

This is a list of supplementary files associated with this preprint. Click to download. 